\title{
Peroxysomal Carnitine Acetyl Transferase Influences Host Colonization Capacity in Sclerotinia sclerotiorum
}

\author{
D. Liberti, ${ }^{1,2}$ J. A. Rollins, ${ }^{1}$ and K. F. Dobinson ${ }^{2,3}$ \\ ${ }^{1}$ Department of Plant Pathology, University of Florida, Gainesville 32611, U.S.A.; ${ }^{2}$ Agriculture and Agri-Food Canada, \\ London, Ontario, N5V 4T3, Canada; ${ }^{3}$ University of Western Ontario, London, Ontario, N6A 5B7, Canada
}

Submitted 12 March 2013. Accepted 27 March 2013.

In lower eukaryotes, the glyoxylate cycle allows cells to utilize two-carbon compounds when simple sugars are not available. In filamentous fungi, glyoxylate metabolism is coupled with $\beta$-oxidation of fatty acids, and both are localized to ubiquitous eukaryotic organelles called peroxisomes. Acetyl coenzyme A (acetyl-CoA) produced during $\beta$-oxidation is transported via the cytosol into mitochondria for further metabolism. A peroxisomal-specific pathway for acetyl-CoA transport requiring peroxisomal carnitine acetyl transferase (CAT) activity has been identified in Magnaporthe grisea peroxisomes. Here, we report that a Sclerotinia sclerotiorum ortholog of the $M$. grisea peroxisomal CAT-encoding gene Pth2 (herein designated Ss-pth2) is required for virulenceassociated host colonization. Null (ss-pth2) mutants, obtained by in vitro transposon mutagenesis, failed to utilize fatty acids, acetate, or glycerol as sole carbon sources for growth. Gene expression analysis of these mutants showed altered levels of transcript accumulation for glyoxylate cycle enzymes. Ss-pth2 disruption also affected sclerotial, apothecial, and appressorial development and morphology, as well as oxalic acid accumulation when cultured with acetate or oleic acid as sole carbon nutrient sources. Although mutants were able to penetrate and initially colonize host tissue, subsequent colonization was impaired. Genetic complementation with the wild-type $S s$-pth2 restored wild-type virulence phenotypes. These findings suggest an essential role in $S$. sclerotiorum for the peroxisomal metabolic pathways for oxalic acid synthesis and host colonization.

Sclerotinia sclerotiorum (Lib.) de Bary is one of the most nonspecific and ubiquitous plant pathogens, causing disease in over 400 species of plants (Boland and Hall 1994). To initiate infection, the $S$. sclerotiorum sclerotia produce inoculum directly through mycelial germination or indirectly through

Nucleotide sequence data for Ss-pth2 is available in the GenBank database under accession number XP_001585822.

Current address for D. Liberti: Nunhems Netherlands B.V., P.O. Box 4005, 6080 AA Haelen, The Netherlands.

Corresponding author: K. F. Dobinson; Telephone: 519-457-1470 X276; Fax: 519-457-3997; E-mail: katherine.dobinson@agr.gc.ca

* The $\boldsymbol{e}$-Xtra logo stands for "electronic extra" and indicates that one supplementary figure, one supplementary table, and supplementary text are published online.

This article is in the public domain and not copyrightable. It may be freely reprinted with customary crediting of the source. The American Phytopathological Society, 2013. carpogenic germination and ascospore production. The fungus usually grows out from a saprotrophic base, imbedded in dead or dying plant tissues, developing into an aggressive, necrotrophic form that penetrates through a healthy plant cuticle (Lumsden and Dow 1973). Unless invasion of the plant occurs through a natural opening (e.g., the stomata) or a wound, mycelia break through the cuticle using a series of modified hyphae that range from nonmelanized simple appressoria to multicellular compound appressoria (infection cushions) (Purdy 1958; Tariq and Jeffries 1984).

In contrast to biotrophic and hemibiotrophic pathogens, which have evolved effector-mediated interactions with their hosts, S. sclerotiorum necrotrophy is thought to be derived from the activity of hydrolytic enzymes, such as cell-wall-degrading enzymes, and production of metabolites, such as oxalic acid, that act as toxins. Oxalic acid is recognized to play a central role in S. sclerotiorum pathogenicity (Cessna et al. 2000; Dutton and Evans 1996; Godoy et al. 1990; Kim et al. 2008; Maxwell and Lumsden 1970; Rollins 2003; Williams et al. 2011), and has a number of physiological roles in pathogenesis, each reported to facilitate plant infection (Bolton et al. 2006). In various fungal species, oxalate biosynthesis has been shown to be associated with the glyoxylate cycle and, specifically, derived by oxidation of glyoxylate (Dutton and Evans 1996). In $S$. sclerotiorum, oxalic acid is generated by oxaloacetate acetyl hydrolase (Maxwell 1973), which hydrolyzes oxaloacetate to produce oxalate and acetate (Dutton and Evans 1996). The primary source of the oxaloacetate may be the TCA cycle, the glyoxylate cycle, or both (Amselem et al. 2011), and the association of the glyoxylate cycle with oxalate biosynthesis in $S$. sclerotiorum remains uncertain.

The glyoxylate pathway catalyzes the conversion of isocitrate to malate and succinate through the activities of isocitrate lyase and malate synthase, and is essential for utilization of twocarbon molecules as carbon sources. This pathway also plays a role in $\beta$-oxidation of fatty acids in many fungal species, and many of the enzymes involved in lipid metabolism are required for pathogenic processes (Lorenz and Fink 2001; Wang et al. 2003). For example, the first enzyme in the pathway, isocitrate lyase, is required for virulence of Magnaporthe grisea, Leptosphaeria maculans, and Colletotrichum lagenarium (Asakura et al. 2006; Wang et al. 2003), and the second enzyme of the pathway, malate synthase, is needed for virulence of Stagonospora nodorum (Solomon et al. 2004). In addition, disabling the lipid catabolic pathway of Sclerotinia sclerotiorum through mutation of the malate synthase gene resulted in defects in pathogenicity when infection was initiated under nutrient-poor conditions (Liberti et al. 2007).

Among the factors that regulate the proper function of the glyoxylate cycle, the intracellular transport of acetyl coenzyme 
A (acetyl-CoA) plays a central role (Kunau et al. 1995; Kurihara et al. 1992; Smith et al. 2000). Recently, a peroxysomal carnitine acetyl transferase (CAT) in M. grisea, Mg-Ptht2, was discovered to facilitate acetyl-CoA transport specifically to the peroxisome, and to be required for both fatty acid $\beta$-oxidation and pathogenicity (Bhambra et al. 2006). To further explore the biological role of fatty acid metabolism in the pathogenicity of $S$. sclerotiorum, we created transposon-disrupted (null) mutants of Ss-pth2, an ortholog of Mg-Pth2, and determined the effects of Ss-pth2 disruption on sclerotial, apothecial, and compound appressoria development, as well as on oxalic acid biosynthesis and disease symptom development.

\section{RESULTS}

\section{Isolation and identification}

of the peroxysomal CAT gene (Ss-pth2) in S. sclerotiorum.

In a BlastP search of the $S$. sclerotiorum genome database (Amselem et al. 2011) with the $M$. grisea Pth2 sequence (accession number AAB88887), six sequences were identified that contained CAT domains. Four of these (SS1G_06923.3, SS1G_ 09446.3, SS1G_07098.3, and SS1G_01807.3) encode multidomain proteins carrying carboxy-terminal choline/CAT domains, while SS1G_13339.3 and SS1G_01589.3 each contain a single CAT domain. Of the latter sequences, SS1G_13339.3 was highly similar (74\% identical at the amino acid level) to the $M$. grisea peroxisomal CAT-encoding Pth2 and, therefore, was designated Ss-pth2, whereas SS1G_01589.3 was highly similar to the Aspergillus nidulans gene encoding the CAT superfamily member FacC (Stemple et al. 1998) (accession number AAC82487). A tblastn (Altschul et al. 1997) search of the National Center for Biotechnology Information (NCBI) database further demonstrated that orthologs of both single CAT-domain genes are found in other filamentous fungi, and the resulting phylogenetic tree (Supplementary Fig. S1) contained two distinct branches corresponding to the Pth2- and FacC-like orthologs, respectively.

Since Ramos-Pamplona and Naqvi (2006) demonstrated that only the Pth2-like CAT is required for plant infection and lipid catabolism in $M$. grisea, we focused our attention on functional analysis of Ss-pth2. To verify the Ss-pth2 (SSIG_13339.3) putative start codon, and locations of the intron/exon junctions, an approximately $2.5-\mathrm{kb}$ sequence of $S s$-pth 2 was amplified from wild-type (WT) strain 274 cDNA. The cDNA sequence was identical to that of SS1G_13339.3 but differed with respect to the location of the start codon, which was 534 nucleotides downstream of that which had been annotated. Alignment of the $S s$-pth 2 cDNA sequence with that of the genomic DNA (gDNA) sequence and of peroxisomal CAT genes from other filamentous fungi (Bhambra et al. 2006; Ramos-Pamplona and Naqvi 2006) confirmed the sequence length and start codon position that we had determined (data not shown). Based on these data, the $S s-p t h 2$ gene comprises a $1.83-\mathrm{kb}$ sequence containing three exons, and a predicted open reading frame encoding a 610 -amino-acid $(69.2-\mathrm{kDa})$ protein with an AKL type I peroxisomal targeting sequence at its $\mathrm{C}$ terminus. Therefore, GenBank accession XP_001585822 was updated with this new annotation.

\section{Ss-pth2 and Ss-mls1 expression in WT S. sclerotiorum.}

To explore the potential function of $S s-p t h 2$ in fatty acid metabolism, we conducted a semiquantitative examination of Ss-pth2 expression during growth of the fungus on media containing two-, three-, or four-carbon (2-, 3-, or 4-C) compounds as sole carbon sources. We also assessed expression of the gene encoding the glyoxylate cycle enzyme malate synthase, which we previously showed to be indispensable for 2-C me-

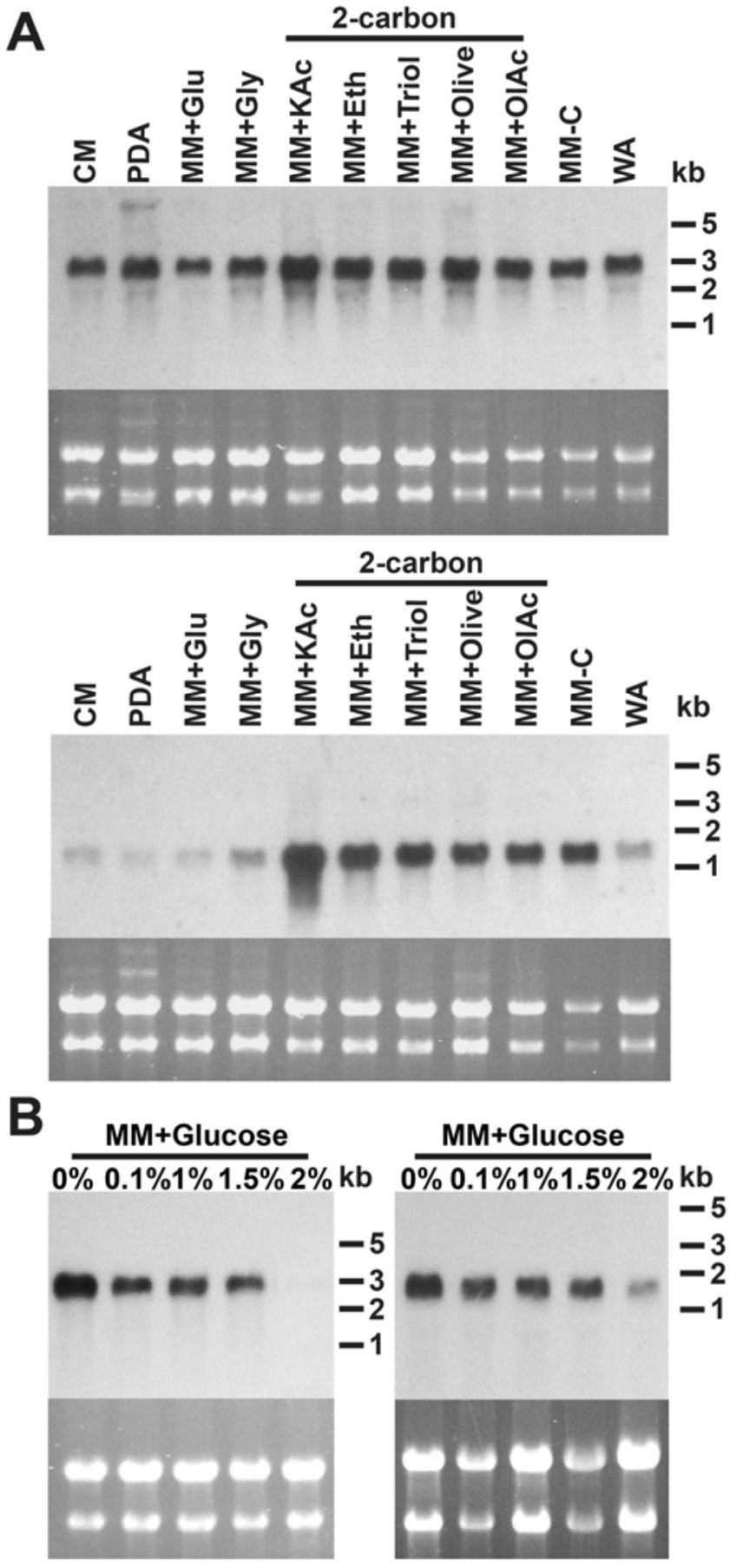

Fig. 1. Northern hybridization analysis of $S s-m l s 1$ and $S s-p t h 2$ expression in Sclerotinia sclerotiorum. Cultures of strain 274 were grown on cellophane-overlaid media in the presence of different carbon sources, and total RNA extracted, fractioned by electrophoresis through a denaturing gel ( $5 \mu \mathrm{g}$ per lane), and blotted onto nylon membranes (Roche Biomolecular Diagnostics). Blots were hybridized with digoxigenin (DIG)-labeled RNA probes. A, Mycelia were grown for 3 days on complete medium (CM), potato dextrose agar (PDA), water agar (WA), or minimal medium (MM) containing $30 \mathrm{mM}$ glucose (MM+Glu), $60 \mathrm{mM}$ glycerol (MM+Gly), 100 $\mathrm{mM}$ potassium acetate (MM+KAc), $60 \mathrm{mM}$ ethanol (MM+Eth), $2.14 \mathrm{mM}$ Triolein (MM+Triol), $2.14 \mathrm{mM}$ olive oil (MM+Olive), $6.6 \mathrm{mM}$ oleic acid (MM+OlAc), or lacking a carbon source (MM-C). Blots were hybridized with $S s$-pth2 (top panel) or Ss-mls1 probes (bottom panel). B, Ss-pth2 and $S s$-mls 1 expression (left and right panels, respectively) in cultures grown on MM lacking glucose $(0 \%)$ or containing $5.5 \mathrm{mM}(0.1 \%), 55 \mathrm{mM}$ $(1.0 \%), 82.5 \mathrm{mM}(1.5 \%)$, or $110 \mathrm{mM}(2 \%)$ glucose. Amounts of ethidiumbromide-stained total RNA loaded into each lane are shown below all panels. Sizes of molecular mass standards (single-stranded RNA molecular marker; Roche Biomolecular Diagnostics) are indicated to the right of each panel. 
tabolism in S. sclerotiorum (Liberti et al. 2007). An approximately 3-kb Ss-pth2 transcript was highly expressed during growth on all tested media (Fig. 1A, top panel). In contrast, elevated expression of the approximately $1.3-\mathrm{kb} S s-m l s 1$ transcript was observed during growth on each of the 2-C sources or on minimal medium (MM) agar lacking a carbon source (MM-C), whereas low levels were observed when the fungus was grown on rich carbon sources (complete medium [CM], potato dextrose agar [PDA], MM + glucose [MM+Glu], and $\mathrm{MM}+$ glycerol) or water agar (WA) (Fig. 1A, bottom panel). Finally, during growth under varying glucose levels (0 to $2 \%$ ), both $S s$-pth 2 and $S s-m l s 1$ expression were at a peak when glucose was absent, whereas only low levels were observed during growth with $2 \%$ glucose (Fig. 1B)

\section{Ss-pth2 disruption and complementation.}

ATMT (Agrobacterium tumefaciens-mediated transformation) was used to generate strains in which the WT Ss-pth2 gene was replaced by the $s s-p t h 2$ (gene disruption) construct in pDL-spth2Tn5 (Fig. 2A). Of 51 putative transformants, 10 retained the ability to grow on selective medium (SM) following mass transfers and one hyphal tip (HT) purification (corre-

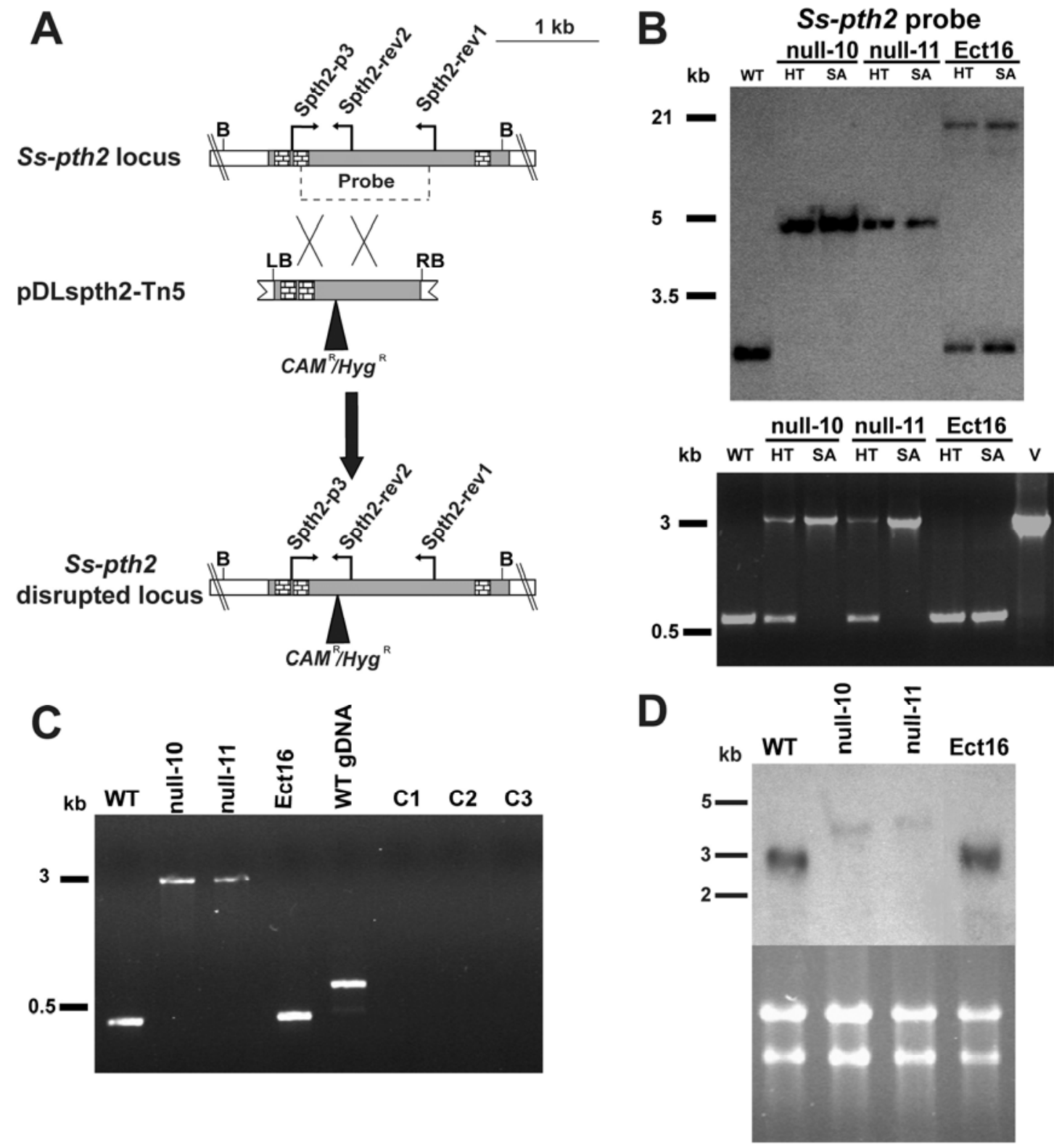

Fig. 2. Targeted disruption of the Sclerotinia sclerotiorum Ss-pth2 gene. A, Schematic representations. Top, the Ss-pth2 (wild-type; WT) locus; middle, the pDLspth2 (Tn5-disrupted) gene construct; bottom, the disrupted locus (ss-pth2) generated by homologous recombination. Coding sequences are shown in gray and introns in a brick pattern. Locations of primers (spth2-p3 and spth2-rev1), the Ss-spth2 hybridization probe (dashed lines), and the approximately 2.5-kb antibiotic resistance gene cassette (arrowhead) are shown above and below the line drawings, respectively. B, DNA blot hybridization and reversetranscription polymerase chain reaction (RT-PCR) analysis of transcription of the WT isolate, and hyphal tip (HT) or single-ascospore (SA) strains purified from transformants SSAT46-10 (null-10), SSAT46-11 (null-11), and SSAT46-16 (Ect16). Top panel, BglII-digested genomic DNA hybridized with Ss-pth2. Bottom panel, PCR amplicons generated, using the spth2-p3/spth2-rev2 primer pair, from genomic DNA from WT, HT, and SA transformants null-10, null11, and Ect16. C, RT-PCR amplification with the spth2-p3/spth2-rev2 primer pair, to assess Ss-pth2 transcription in the WT and null-10, null-11, and Ect16 SA transformants. WT genomic DNA (gDNA), C1, C2, and C3 (null-10, null-11, and Ect16 gDNA, respectively) are negative control templates. D, RNA blot hybridization. RNA from the WT and SA null-10, null-11, and Ect16 hyphae. Sizes of molecular mass standards are indicated to the left in B, C, and D: digoxigenin (DIG) III molecular markers (Roche Biomolecular Diagnostics) (B, top); 1 kb Ladder Marker (NEB) (B, bottom, and C); and single-stranded RNA molecular markers (Roche Biomolecular Diagnostics) (D). 
sponding to a transformation frequency of 10 per $10^{5}$ ascospores). Southern blot hybridization analysis demonstrated that five transformants (SSAT46-10A [null-10], SSAT46-11A [null11], SSAT46-12A, SSAT46-45A, and SSAT46-51C) had the WT gene replaced by the gene disruption construct whereas, in four transformants (SSAT46-16A [Ect16], SSAT46-41, SSAT46-43, and SSAT46-50A), the construct was ectopically integrated into the genome (Fig. 2B, top panel; data not shown).
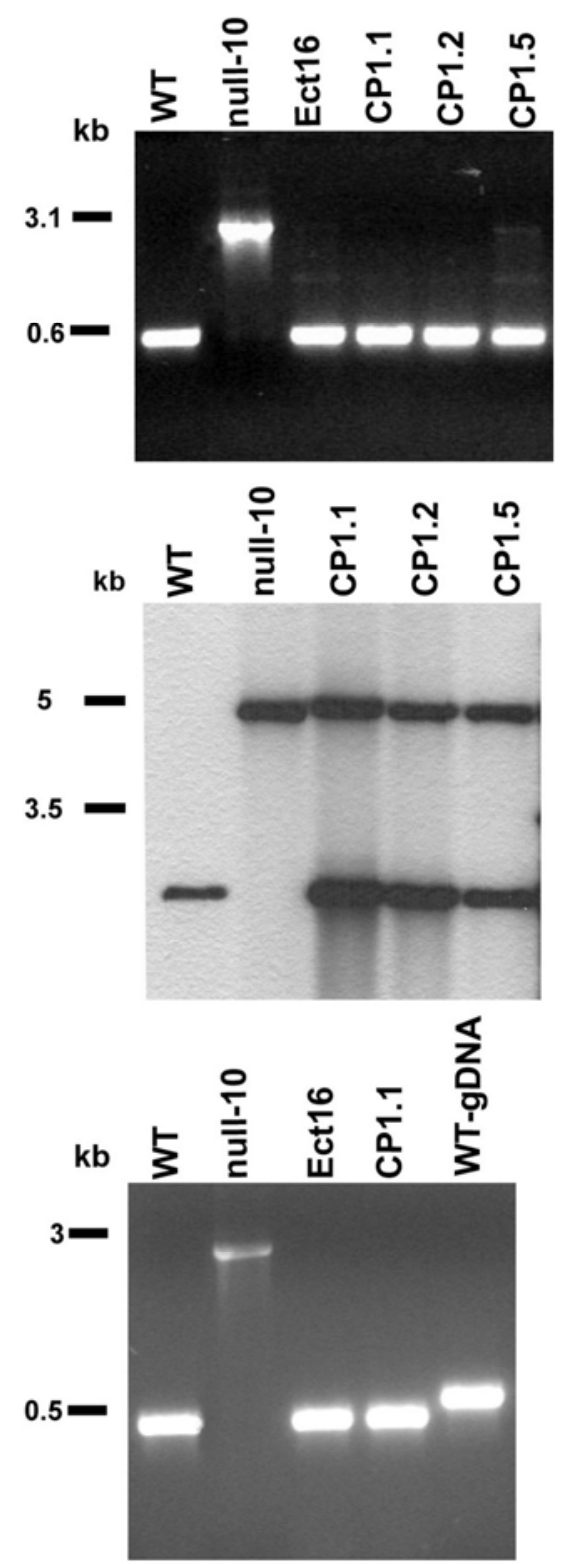

Fig. 3. Molecular identification of Ss-pth2-complemented strains. Southern hybridization and polymerase chain reaction (PCR) analyses were used to compare complements of Ss-pth2and ss-pth2 alleles in wild-type (WT; Sspth2) isolate 274 with that in single-ascospore (SA) strains derived from null-10 (SSAT46-10A), Ect16, or complemented strains CP1.1, CP1.2, and CP1.5. Top: The spth2-p3/spth2-rev2 primer pair was used to synthesize PCR amplicons from genomic DNA of all strains. Middle: DNA blot hybridization. BglII-digested DNA was hybridized with a digoxigenin (DIG)-labeled Ss-pth2 gene probe. Bottom: Reverse-transcription PCR amplification used the spth2-p3/spth2-rev2 primer pair to assess Ss-pth2 expression. Strains were grown on cellophane-overlaid complete medium agar, and RNA was isolated with TRIzol Reagent (Invitrogen). Sizes of molecular mass standards are indicated to the left of each panel (top and bottom: 1-kb Ladder Marker (NEB); middle: DIG III molecular markers [Roche Biomolecular Diagnostics]). Ethidium bromide staining of the gels confirmed that equivalent amounts of sample RNAs were loaded onto the gels (data not shown).
Two mutants, null-10 and null-11, as well as transformant Ect16 (which contains the WT gene and an ectopically integrated copy of the mutant gene), were single-ascospore (SA) purified. Polymerase chain reaction (PCR) analysis of HT and SA strains confirmed that the SA but not HT strains of null-10 and null-11 carried only the mutant gene (ss-pth2), whereas amplicons produced from SA transformant Ect16 confirmed the presence of a copy of the WT Ss-pth2 (Fig. 2B, bottom panel). SA cultures of null-10, null-11, and Ect16 were used for all further experiments.

To verify that the WT $S s$-pth2 transcript was not being expressed in ss-pth2 strains, reverse-transcription (RT)-PCR analysis of the mutant and WT strains was performed, reveal-

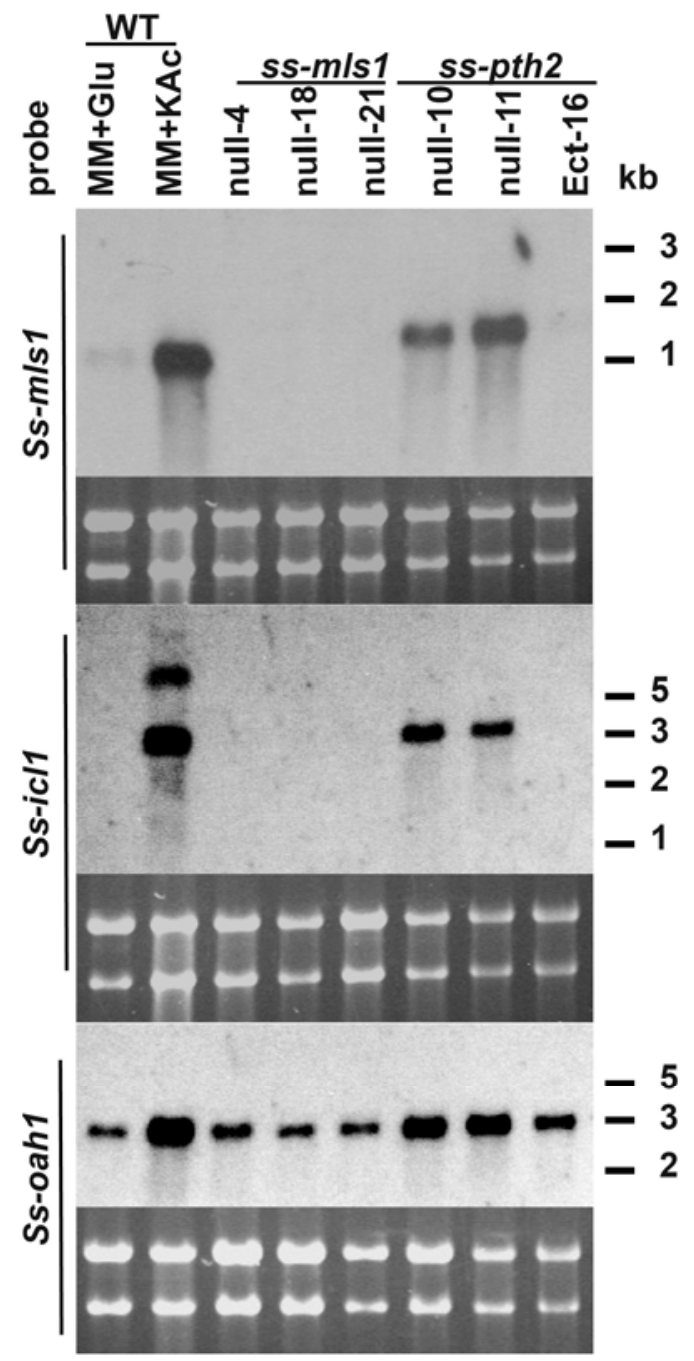

Fig. 4. Northern blot analysis of transcription from Sclerotinia sclerotiorum malate synthase, isocitrate lyase, and oxaloacetate acetyl hydrolase genes (Ss-mls1, Ss-icll, and $S s$-oahl, respectively) in wild-type (WT) and single-ascospore (SA) strains of $\mathrm{ss}$-mls 1 null mutant transformants SSAT42-14A, SAT42-18B, and SAT42-21A (null-4, null-18, null-21), sspth2 null mutants SSAT46-10A, and SAT46-11A (null-10, and null-11), and SSAT46-16A (Ect16). RNA was extracted from WT cultures grown for 3 days at room temperature on cellophane-overlaid-minimal medium agar containing $30 \mathrm{mM}$ glucose $(\mathrm{MM}+\mathrm{Glu})$ or $100 \mathrm{mM}$ potassium acetate (MM+KAc). RNA from transformants was extracted from cultures grown for 3 days as above, on MM+Glu agar. Blots were hybridized with the indicated gene-specific probes $(S s-m l s 1, S s$-icll, and $S s$-oah1). Quality and amount of RNA loaded into the corresponding lanes of each gel was assessed by ethidium bromide staining, as shown in the photograph directly below each blot. Sizes of molecular mass standards (single-stranded RNA molecular marker; Roche Biomolecular Diagnostics) are indicated to the right of each panel. 
ing that amplification from WT RNA produced an amplicon of the expected $0.5 \mathrm{~kb}$ while RNA of the mutants produced an approximately 3.1-kb amplicon (Fig. 2C). Northern hybridization also showed that the WT strain produced a 3-kb transcript (the anticipated size), whereas both mutants lacked the WT transcript but accumulated very low levels of an approximately 4-kb transcript (Fig. 2D). The results of further RTPCR analyses indicated that the approximately 4-kb RNA detected in the null mutants is a read-through of the chloramphenicol-resistant and hygromycin B-resistant $\left(\mathrm{CAM}^{\mathrm{r}} / \mathrm{Hyg}^{\mathrm{r}}\right)$ cassette (data not shown).

Ss-pth2-complemented strains (CP1.1, CP1.2, and CP1.5) were produced by transformation of the SA mutant null-10 with vector pDL103 containing the Ss-pth2 gene with its endogenous promoter. PCR analysis indicated that the nourseothricintagged Ss-pth2 gene had integrated into the null-10 genome (Fig. 3, top panel), and Southern hybridization verified that the complementation construct was integrated ectopically into the genome of each transformant (Fig. 3, middle panel). One strain, CP1.1, was SA purified, and WT transcript accumulation confirmed by RT-PCR (Fig. 3, bottom panel).

\section{Ss-pth2 disruption alters expression}

of glyoxylate cycle genes $S s-m l s 1$ and $S s-i c l 1$

and the oxalate biosynthetic gene $S s$-oah1.

Accumulation of Ss-mls1, Ss-icll, and Ss-oahl transcripts was assessed semiquantitatively by Northern hybridization using WT, ss-mls1-null, and ss-pth2-null strains. The WT strain accumulated $S s-m l s 1, S s$-icll, and $S s$-oahl transcripts when growing on $\mathrm{MM}+$ potassium acetate $(\mathrm{MM}+\mathrm{KAc})$ but exhibited little $(S s-o a h 1)$ or no $(S s-m l s 1$ and $S s$-icll) transcript accumulation when grown on MM+Glu. Growing on MM+Glu, the $s s-m l s 1$ mutants (null-4, null-18, and null-21) obtained from a previous study (Liberti et al. 2007) and the Ss-pth2 ectopic transformant (Ect-16) behaved like the WT (Fig. 4). In contrast, the $s s-p t h 2$ strains (null-10 and null-11) exhibited a high level of accumulation for $S s-m l s 1, S s$-icll, and $S s$-oahl when cultured on MM+Glu (Fig. 4).

\section{Morphological defects in ss-pth2 mutants.}

A surprising feature was the absence of sclerotial development when the null mutants were grown on HygB-amended (selective) medium. In the absence of the selection pressure, sclerotial production was comparable with that of the WT parent (Fig. 5A; Table 1; data not shown). Moreover, sclerotia produced without selection by the null-10 and null-11 strains were able to produce apothecia when conditioned and incubated for carpogenic germination. Apothecia differed from those of WT, with short stipes and disks that did not fully expand (Fig. 5B; data not shown). Additionally, ascospore germination was slightly reduced in the null strains (Table 1). Defects in sclerotia development, apothecia morphology, and ascospore germination were restored in CP1.1 and other complemented strains (Fig. 5A; Table 1; data not shown).

Compound appressorium development in the ss-pth $2 \mathrm{mu}-$ tants was assessed both macroscopically and microscopically and shown to be aberrant. The null ss-pth2 strains grown on cellophane produced up to twofold more mature compound appressoria than did the WT strain (Table 1), as shown by the number of pigmented, aggregated hyphae (Fig. 5C, left). Such overproduction was observed on various hydrophobic surfaces, such as Parafilm and plastic petri dishes (data not shown). Microscopic examination of the hyphal branching pattern of mycelia grown on cellophane further revealed that compound

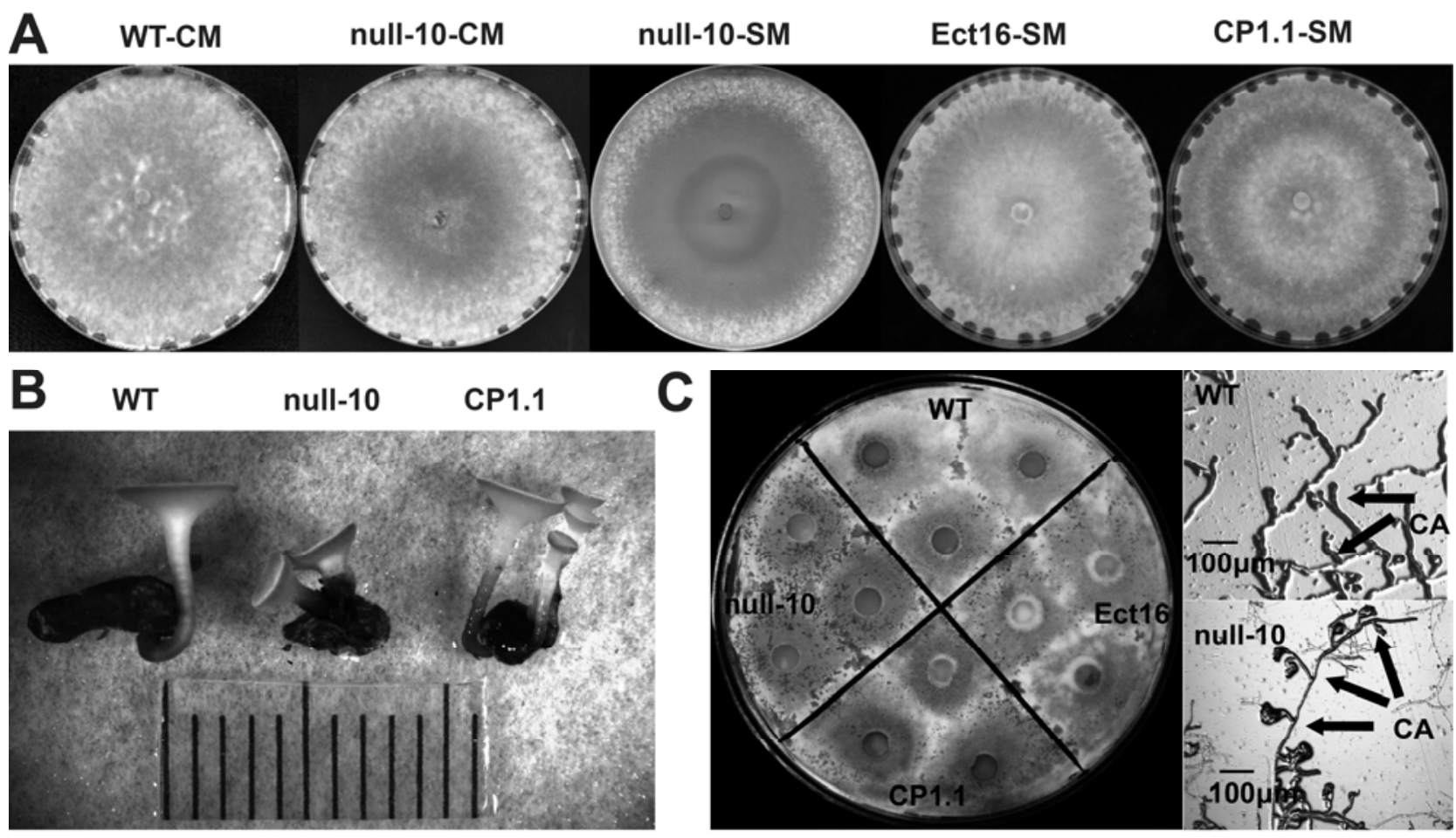

Fig. 5. Effects of Ss-pth2 disruption on sclerotial, apothecial, and compound appressorial morphologies. A, Sclerotial phenotypes of wild-type (WT) and transformant SSAT46-10A (null-10) grown on complete medium (CM) agar, and of null-10 and transformants SSAT46-16A (Ect16) and SSAT-7.8.09-P1.1 (CP1.1) grown on selective medium (SM; CM agar containing Hygromycin B). All transformants were single-ascospore-purified. B, Apothecial phenotypes of WT, null-10, and CP1.1. Ruler $=1.1 \mathrm{~cm}$. C, Compound appressoria (CA) produced by WT, null-10, Ect16, and CP1.1. Macroscopic (left panel) and microscopic (right panels) views. Cultures were grown on potato dextrose agar overlaid with cellophane. In the macroscopic view (left panel) and micrographs (right panels), CA were easily identified on newly formed vegetative hyphae from both the null strain and WT; arrows in the left and right panels indicate pigmented aggregates and hyphal branches developing into CA initials, respectively. 
appressoria were present at a high frequency at each hyphal branch in the ss-pth2 mutants (Fig. 5C). Microscopic examinations were subsequently made of mycelia-inoculated onion epidermal strips and, at $16 \mathrm{~h}$ postinoculation (hpi), both mutants and the WT were producing compound appressoria, penetrating the onion epidermis, and producing infection hyphae (data not shown).

\section{Effects of Ss-pth2 disruption}

on growth, pathogenicity, and oxalate accumulation.

The null ss-pth2 strains grew normally on PDA whereas, starting from $36 \mathrm{hpi}$, a reduction was noticeable in radial growth on MM+Glu agar compared with that of WT, Ect-16 transformant (ectopic), and complemented strains (Fig. 6A). In addition, the ss-pth2 mutants failed to grow on MM amended with glycerol, potassium acetate, oleic acid, or glycerol as sole carbon sources, and growth was restored when $s s-p t h 2$ strain null-10 was genetically complemented (CP1.1) (Fig. 6B).

When MM+Glu- and PDA-grown ss-pth2 strains were inoculated onto unwounded, detached soybean leaflets, we observed smaller necrotic lesions relative to those produced by WT, ectopic, and complemented strains (Fig. 7). Although, at 48 hpi, no differences in size were noticeable between lesions generated by the $s s$-pth 2 and WT strains (all were $<5 \mathrm{~cm}^{2}$ ), subsequent to $48 \mathrm{hpi}$, the expansion rate of ss-pth2 strain lesions was much slower than that of lesions generated by the WT, ectopic, and complemented strains (Fig. 7A to D). This difference was nutrient dependent, with slightly more and faster expansion from

Table 1. Phenotypes of Sclerotinia sclerotiorum strains used in this study ${ }^{\mathrm{a}}$

\begin{tabular}{llcc}
\hline Strain & \multicolumn{1}{c}{$\begin{array}{c}\text { Genotype } \\
\text { (antibiotic resistance) }\end{array}$} & $\begin{array}{c}\text { Germinating } \\
\text { ascospores }(\%)\end{array}$ & $\begin{array}{c}\text { Sclerotia } \\
\text { (number per plate) }\end{array}$ \\
\hline 274 & Ss-pth2 & $98.8 \pm 1.2$ & $30.5 \pm 3.1$ \\
null-10 & Compound appressoria \\
null-11 & ss-pth2 $\left(\mathrm{Hyg}^{\mathrm{r}}\right)$ & $91.5 \pm 3.5$ & $28.2 \pm 2.7$ \\
Ect16 $^{\mathrm{c}}$ & ss-pth2 $\left(\mathrm{Hyg}^{\mathrm{r}}\right)$ & $89.2 \pm 4.5$ & $31.5 \pm 4.4$ \\
CP1.1 $^{\mathrm{d}}$ & Ss-pth2, $s s-p t h 2\left(\mathrm{Hyg}^{\mathrm{r}}\right)$ & $95.5 \pm 5.6$ & $28.4 \pm 2.3$ \\
\hline
\end{tabular}

${ }^{\text {a }}$ Average \pm standard deviation $(n=9$; three experiments with three replicates/experiment).

${ }^{\text {b }}$ Hygromycin-resistant $\left(\mathrm{Hyg}^{\mathrm{r}}\right)$ transformant of parental strain 274 with $S$ s-pth2 replaced by the $S s$-pth2 disruption construct.

${ }^{\mathrm{c}} \mathrm{Hyg}^{\mathrm{r}}$ transformant of strain 274 carrying an ectopic copy of the Ss-pth2 disruption construct.

d Transformant of the null-10 strain, carrying nourseothricin resistance (Nour ${ }^{\mathrm{r}}$ ) gene-tagged ectopic copy of Ss-pth2. Ascospore germination was assayed on potato dextrose broth at $24 \mathrm{~h}$ post inoculation. Compound appressoria formation was assayed on cellophane-overlaid potato dextrose agar. Sclerotia production was evaluated from cultures grown on complete medium agar.

A

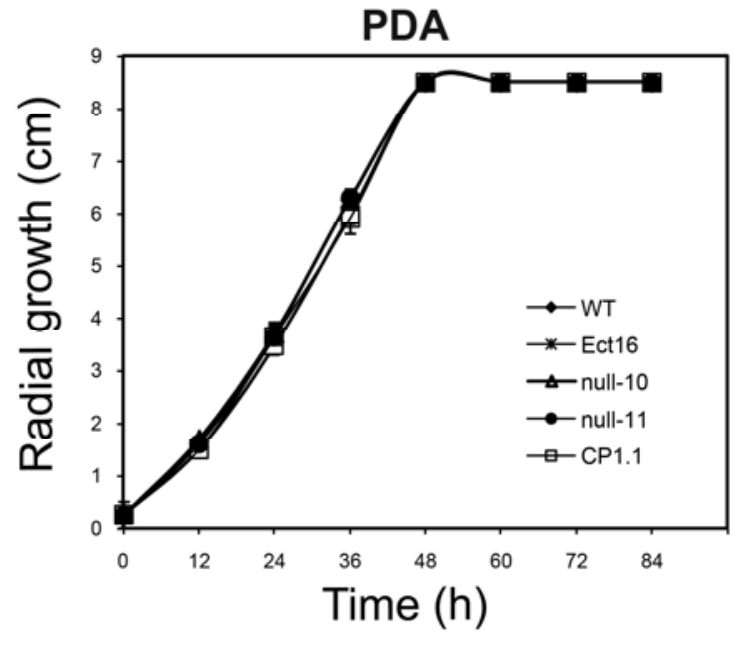

B

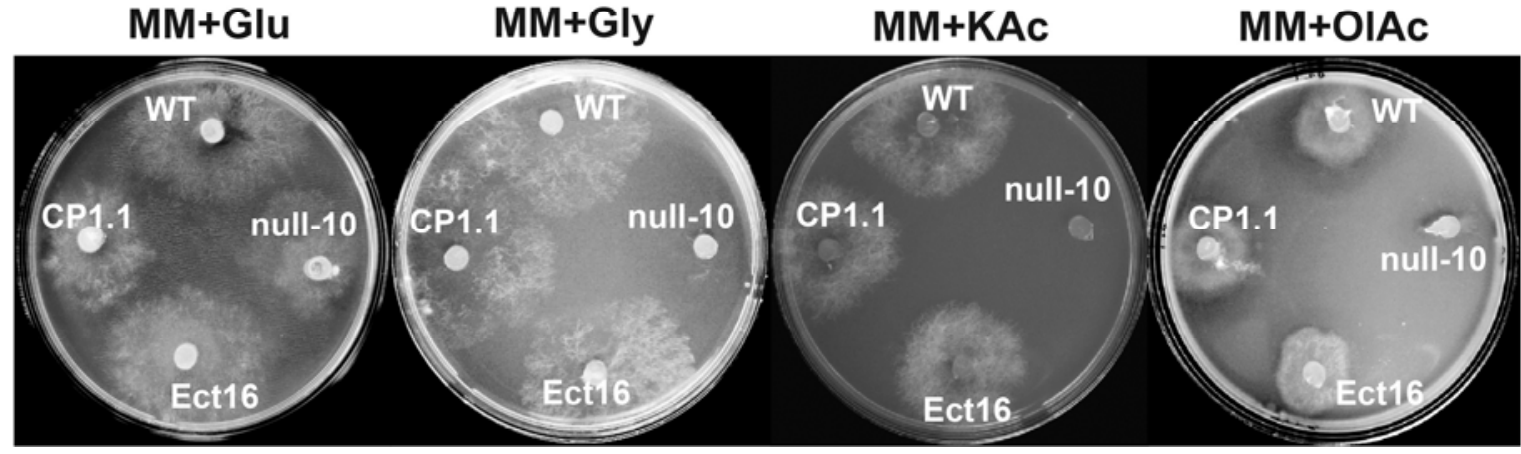

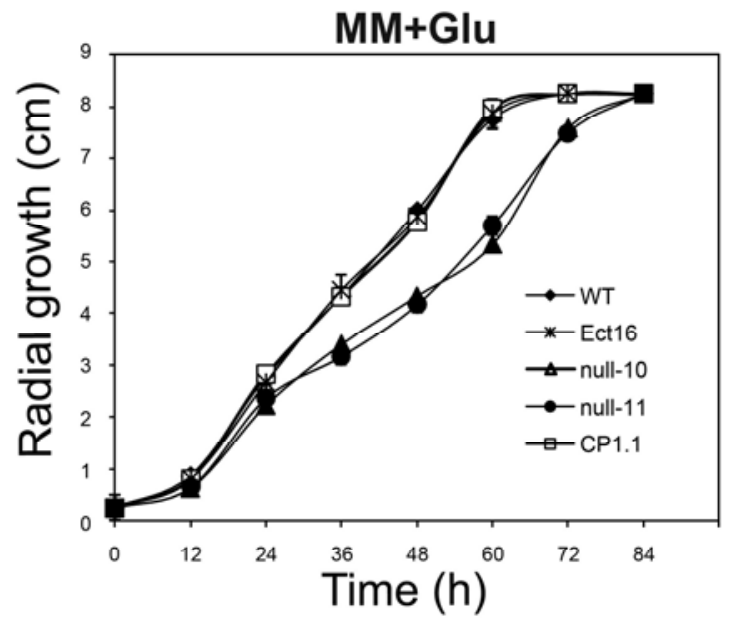

MM+KAc $\quad M M+O I A c$

Fig. 6. Effects of Ss-pth2 disruption on mycelial growth. A, Wild-type (WT) strain 274, ss-pth2 disruption mutants null-10 and null 11 , Ect16 (containing an ectopic copy of the ss-pth2 disruption construct), and complemented strain CP1.1 were grown on potato dextrose agar (PDA) or minimal medium (MM) agar containing $30 \mathrm{mM}$ glucose (MM+Glu), and colony diameters were measured at 12-h intervals. Each point represents the mean colony diameter \pm the standard deviation (the experiment was done twice, with three replicate cultures of each strain used in each experiment). B, Strains were subcultured from MM lacking carbon onto MM+Glu or MM containing $60 \mathrm{mM}$ glycerol (MM+Gly), $100 \mathrm{mM}$ potassium acetate (MM+KAc), or 6.6 mM oleic acid (MM+OlAc). Photographs were taken after 3 days' growth at room temperature on MM+Glu, MM+Gly, and MM+OlAc and after 10 days' growth at room temperature on $\mathrm{MM}+\mathrm{KAc}$. 
PDA-grown inocula than from MM+Glu-grown inocula (Fig. 7A and $\mathrm{B}$, respectively), a difference that was not abrogated by wounding of the leaf (Fig. 7C and D, respectively).

By 72 hpi, leaves infected by the WT strain showed, in addition to significant maceration of host tissue, multiple watersoaked microscopic lesions forming in the healthy portion of the leaf which, after $96 \mathrm{~h}$, became confluent and covered the entire leaf (data not shown). The ectopic strain produced several secondary water-soaked lesions, accompanied by intensive leaf yellowing, that exhibited a delay in becoming confluent, while virulence was fully restored to WT in the CP1.1 strain (Fig. 7E). In contrast, microscopic water-soaked lesions were not produced by the null mutants, even when the mycelial mat continued to grow on the healthy portion of the leaf (data not shown). In addition, soybean leaflets inoculated with these strains displayed a strong defense response, identifiable as reddish-brown lesions surrounded by yellow margins (Fig. 7E). The mutant strains never fully colonized the leaf and, whereas lesions produced by WT and complemented strains were fully expanded after 2 weeks, with sclerotial initials visible, the mutants showed none (Fig. 7E).

In vitro oxalic acid accumulation was evaluated for WT and transformed strains grown for up to $25 \mathrm{~h}$ in MM-MOPS ( $\mathrm{pH} 7$ ) either lacking a $\mathrm{C}$ source or containing glucose, glycerol, potassium acetate, or oleic acid as a sole carbon source. In MM+ Glu medium, oxalic acid gradually accumulated in all strains throughout the experiment (Fig. 8A), whereas in MM+KAcgrown cultures, the gradual accumulation of oxalic acid was preceded by a 5-h lag in production (Fig. 8B), and cultures grown in MM amended with equimolar amounts of glucose
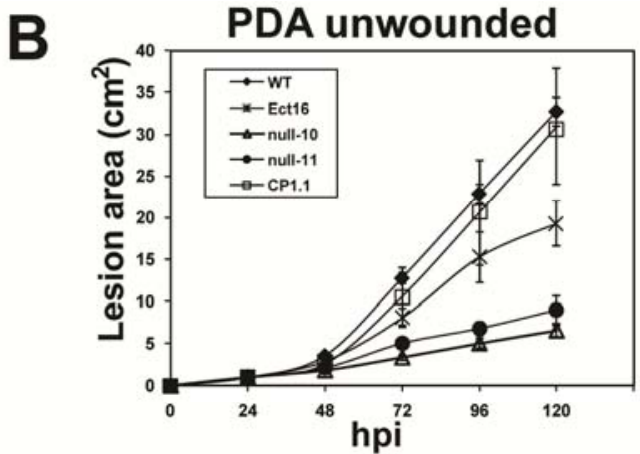

D

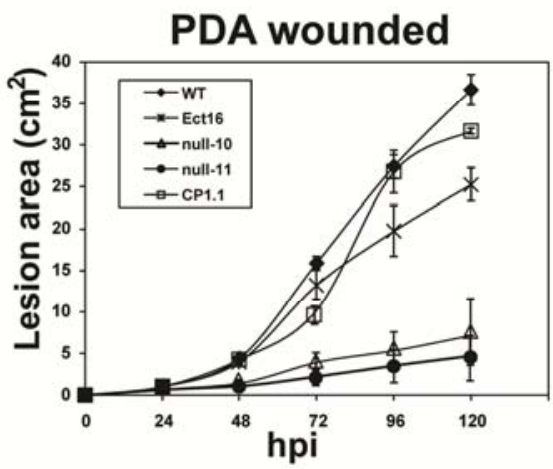

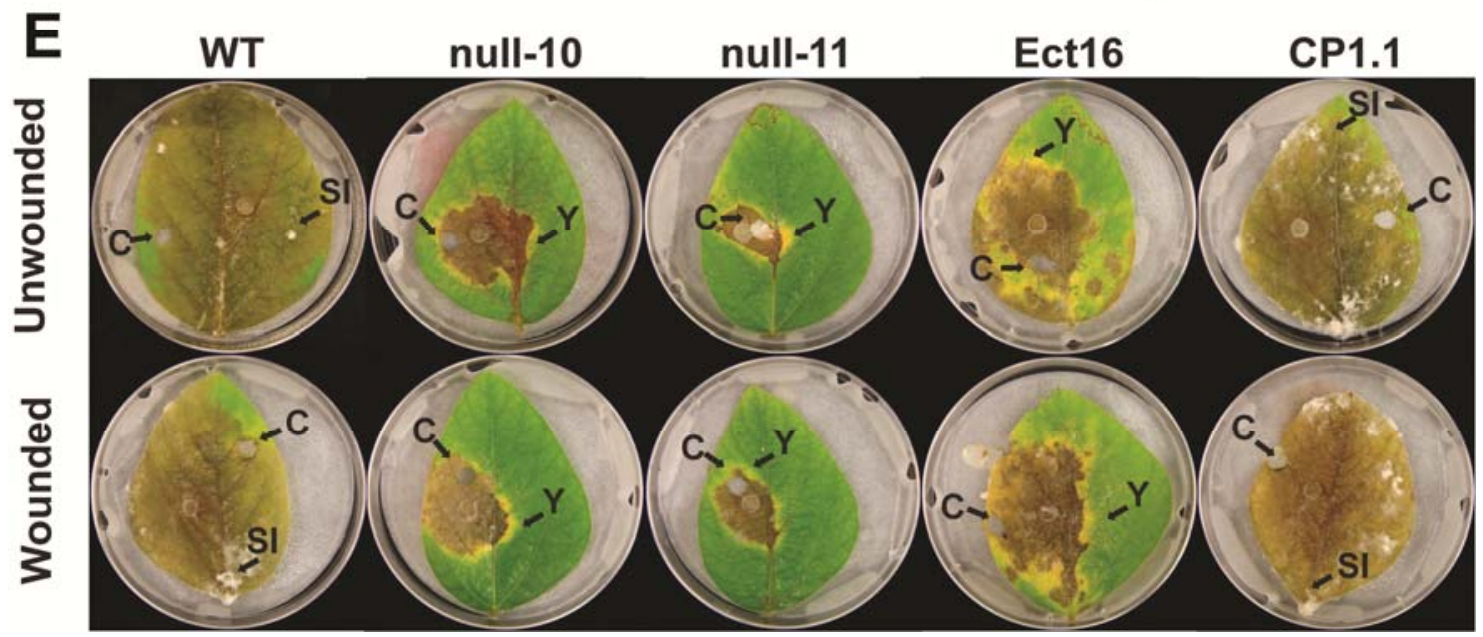

Fig. 7. Pathogenicity and in planta radial growth of wild-type (WT) and transformed strains of Sclerotinia sclerotiorum. A to D, Mean lesion area measured at 24-h intervals on $\mathbf{A}$ and $\mathbf{B}$, unwounded or $\mathbf{C}$ and $\mathbf{D}$, wounded leaves. Leaves were inoculated with plugs of mycelia grown on minimal medium containing glucose $(\mathrm{MM}+\mathrm{glu})$ agar (A and C) or potato dextrose agar (PDA) (B and D). Data points represent the mean and standard deviation of three independent experiments. E, Soybean leaves were inoculated with agar plugs from PDA-grown cultures of WT, ss-pth2 disruption strains (null-10, null-11), the ectopic strain (Ect16), and complemented strain CP1.1. The experiment was repeated four times, and data shown are from one representative experiment. Y: leaf yellowing/discoloration; SI: sclerotial initials; C: site at which 5-mm-diameter leaf plugs were removed for oxalate assay. 
and potassium acetate showed a steep increase in oxalic levels, reaching a plateau by $15 \mathrm{~h}$ (Fig. 8C), similar to that seen during growth on YSPu medium (Rollins 2003). WT and the complemented strain CP1.1 produced oxalate at 1 and 0.6 $\mathrm{mg} / \mathrm{ml}$, respectively, even in the absence of a $\mathrm{C}$ source, and all $\mathrm{C}$ sources tested supported accumulation of high levels of oxalate by WT (approximately $2 \mathrm{mg} / \mathrm{ml}$ ), and moderate levels by $\mathrm{CP} 1.1$ (1.2 to $2 \mathrm{mg} / \mathrm{ml}$ ) (Fig. $8 \mathrm{~A}$ to F). In contrast, oxalate production by the null mutant was lacking during growth with KAc or in the absence of a C source (Fig. 8B and F) and negligible (up to only approximately $0.2 \mathrm{mg} / \mathrm{ml}$ ) during growth with the 2-C source oleic acid (Fig. 8D), whereas growth in media containing either glycerol or glucose resulted in low to moderate levels ( 0.9 to $1.3 \mathrm{mg} / \mathrm{ml}$ ) (Fig. 8A and E).

Finally, an analysis of in vivo oxalic acid accumulation showed that, on both unwounded and wounded leaflets, oxalate production by the null mutants was only approximately $20 \%$ lower than that of the WT, ectopic, and complemented strains (Fig. 8G), a difference that was not statistically significant.

\section{DISCUSSION}

S. sclerotiorum is a necrotrophic plant pathogen which infects and colonizes a large and diverse number of hosts and tissues within these hosts. Such broad-ranging capabilities require the metabolic capacity and flexibility to successfully adapt to variable nutrient conditions. An understanding of these capacities could provide insight into the determinants of host range and necrotrophic aggressiveness. With this goal in mind, the aim of the present investigation was to determine the contribution of peroxisomal CAT activity on S. sclerotiorum growth, development, and pathogenicity.
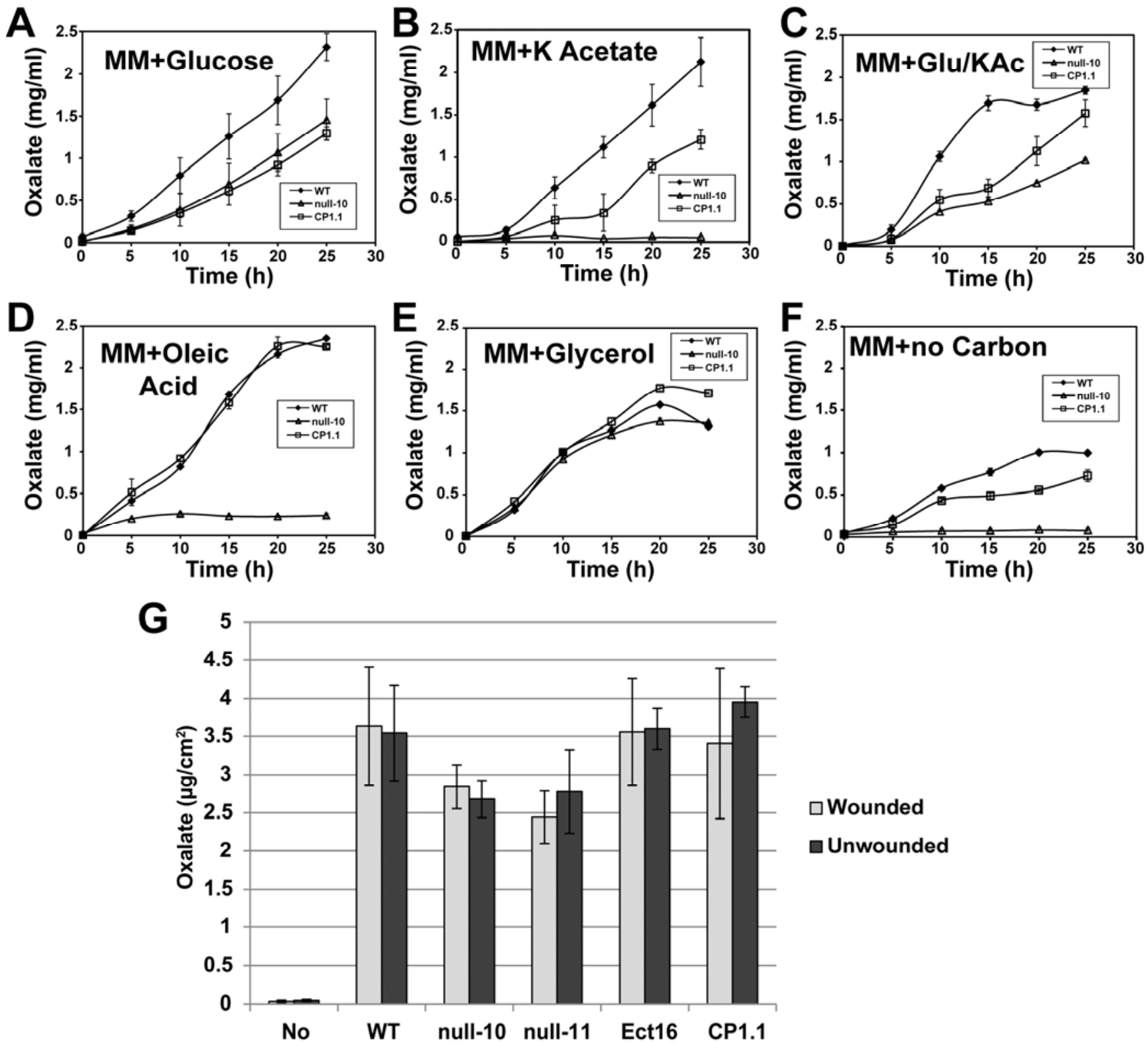

\section{$\square$ Wounded}

$\square$ Unwounded

Fig. 8. Effects of $S s$-pth 2 disruption on oxalic acid accumulation in vitro and in vivo. Oxalic acid accumulation kinetics of wild-type (WT), ss-pth2 mutant (null-10), and ss-pth2-complement (CP1.1) strains cultured in 0.5 M MOPS-buffered minimal medium (MM; pH 7) containing A, 30 mM glucose; B, 100 $\mathrm{mM}$ potassium acetate (K Acetate); C, $30 \mathrm{mM}$ glucose plus $100 \mathrm{mM} \mathrm{K}$ acetate (KAc); D, $6.6 \mathrm{mM}$ oleic acid; E, $60 \mathrm{mM}$ glycerol; or F, no carbon source. Data were pooled from two independent assays (three replicates/strain/assay), with each data point representing the mean \pm standard error. G, In vivo oxalic acid accumulation by WT, null-10, null-11, Ect16, and CP1.1 strains. At 5 days postinoculation, oxalate levels were measured from 5-mm-diameter leaf plugs taken from margins of lesions on the wounded and unwounded soybean leaves. 
The Ss-pth2 gene encodes an ortholog of the Pth2-proteinencoding gene $M g$-Pth2, which is required in $M$. grisea for fatty acid metabolism and pathogenicity (Bhambra et al. 2006). In agreement with previous findings (Bhambra et al. 2006; Ramos-Pamplona and Naqvi 2006; Strijbis et al. 2008, 2010), our functional analyses of Ss-pth2 demonstrated that the gene is indispensable for growth on 2-C sources and is necessary for peroxisome-mitochondrial transport of acetyl units produced during fatty acid $\beta$-oxidation. In addition to the pleotropic metabolic effects that impacted oxalic acid production and, ultimately, pathogenicity, disruption of $S s$-pth2 resulted in defective morphological development.

Both glucose and the nonfermentable 3-C glycerol feed into the glycolytic cycle, and loss of Ss-pth2 gene function resulted in no growth on glycerol and reduced growth on glucose. The latter is not without precedence; Cryptococcus neoformans mutants that lack functional peroxisomes exhibit a reduced growth rate in the presence of glucose (Idnurm et al. 2007). The involvement of Ss-pth2 in both glycerol catabolism and morphological development is intriguing. Bhambra and associates (2006) reported that application of hyperosmotic concentrations of glycerol to $M$. grisea did not reveal a significant difference in appressorial turgor between $\Delta p t h 2$ and WT strains. This finding indicates a lack of Pth2 involvement in generation or maintenance of $M$. grisea appressorium turgor, processes that are dependent on glycerol accumulation within the appressorium (de Jong et al. 1997). In contrast, the S. sclerotiorum ss-pth2 null mutant, which fails to metabolize glycerol for growth, overproduces compound appressoria. Perhaps, rather than playing a role in generating turgor, a functional glyoxalate pathway may be required to metabolize reserves or provide precursors for endogenous developmental regulators in S. sclerotiorum.

Sclerotia of $S$. sclerotiorum are thought primarily to use stores of carbohydrates rather than lipids to support carpogenic germination (Coley-Smith and Cooke 1971) and, although apothecia were formed by the ss-pth2 mutant with a similar timing and number as the WT, these structures were deformed. Therefore, we hypothesize that the developmental phenotypes we observed result from an inability to process fatty acids and, also, lack of catabolic products, endogenous fatty acids, or glycerol. In particular, the inability to fully assimilate endogenous triacylglycerol could disrupt endogenous oxylipin balance or secondary messengers such as diacylglycerol which are known to regulate developmental differentiation in fungi (Noverr et al. 2003; Thines et al. 1997). Taken together, our data suggest that the defect in fatty acid metabolism plays a regulatory role rather than a nutritional role in the observed apothecial morphology phenotype.

The results of this study have begun the process of genetically dissecting the metabolic pathways that participate in $S$. sclerotiorum oxalate biosynthesis. Our findings indicate a remarkable metabolic flexibility in the WT (Ss-pth2) fungus with respect to the production of oxalic acid, the pathogen's primary characterized pathogenicity factor. Consistent with the report of Culbertson and associates (2007), which demonstrated that carbon sources other than glucose can augment $S$. sclerotiorum glucose-dependent growth and regulate oxalate accumulation, we have shown that the WT strain is quite able to grow on and metabolize 2-C and fatty acid-based $\mathrm{C}$ sources for in vitro oxalate production, and produce a low level of oxalate even under severe starvation conditions (lacking a $\mathrm{C}$ source).

The ss-pth2 strain is able to produce near-WT levels of oxalate during growth on either glucose or glycerol, indicating that, in S. sclerotiorum, metabolism of these C sources must occur primarily through the glycolytic pathway and TCA cycle, to generate the oxaloacetate from which oxalate is directly produced. In contrast to the near-WT levels of oxalate pro- duced by the ss-pth 2 mutant in the presence of glycerol despite there being no detectable biomass production, the mutant showed a severe lack of both growth and oxalate production when grown in the absence of a $\mathrm{C}$ source or presence of KAc or oleic acid (2-C and fatty acid $\mathrm{C}$ sources, respectively). This lack of oxalate accumulation could be explained by an inability to metabolize endogenous residual carbon sources, most probably fatty acids, which are known to accumulate as lipid bodies in the hyphal cells (Lösel and Sancholle 1996). Likewise, the inability of the $s s-p t h 2$ strain to use KAc or oleic acid for either growth or oxalate production suggests that the mutant is not able to metabolize either of these $C$ sources or the endogenous carbon sources. Furthermore, although reintroduction into the mutants of the Ss-pth2 gene rescued the developmental defects and restored growth on both $2-\mathrm{C}$ and fatty acid carbon sources, oxalic acid production in complemented strain CP1.1 was not restored to WT levels when grown on those C sources. Potentially, under growth conditions favoring maximal oxalic acid accumulation, ectopic expression of $S s-p t h 2$ is insufficient to fully restore WT metabolism.

In the brown rot wood-decay basidiomycete Fomitopsis palustris, oxalic acid biosynthesis appears to occur through a coupling of the TCA and glyoxylate cycles with associated acetate recycling (Munir et al. 2001). Within these coupled cycles, activities of the glyoxylate enzymes isocitrate lyase and malate synthase were shown to be constitutive and not subject to glucose repression (Munir et al. 2001; Sakai et al. 2006). In contrast to $F$. palustris, the $S$. sclerotiorum glyoxylate cycle genes $S s-p t h 2, S s-i c l 1$, and $S s-m l s 1$ are glucose repressible. In addition, and in keeping with the possibility that the glyoxylate cycle is required for oxalic acid biosynthesis only when utilizing 2-C substrates as sole carbon sources, $S s-p t h 2, S s-m l s 1$, and Ss-icll show high levels of expression during growth of the WT S. sclerotiorum on KAc, and expression also in the $s s-p t h 2$ mutant background. Thus, our data indicate that the glyoxylate and oxalate biosynthetic pathways are tightly coupled in $S$. sclerotiorum, as they are in $F$. palustris, but that the $S$. sclerotiorum glyoxylate cycle is subject to the same regulatory controls as those found in most other fungi (Amor et al. 2000; De Lucas et al. 1994; López et al. 2004; Sakai et al. 2006).

In various pathosystems, it has been demonstrated that, during plant penetration, the pathogen uses reserve carbon sources such as trehalose, glycogen, and lipid bodies for growth and infection structure formation (Solomon et al. 2003). It is possible that the attenuated virulence of the ss-pth 2 mutants could be ascribed, in part, to infection cushion dysfunction resulting from the lack of catabolism of endogenous lipid bodies. However, although the ss-pth2 mutants hyperaccumulated compound appressoria, full virulence was not restored by wounding of the host plant, suggesting that the virulence defect was not due simply to a penetration defect but also to defects in subsequent in planta metabolic processes that led to attenuated host colonization.

Because MM supported slower growth of the mutant than of WT, whereas PDA-grown colonies showed no such growth difference, a defect in lesion expansion might have been expected for the MM-grown mutant. However, the mutant exhibited reduced virulence when either MM- or PDA-grown mycelia were used as inoculum. Therefore, these data support the conclusion that reduced virulence was not attributable to lack of vigor of the initial inoculum but, rather, to subsequent in planta processes, and the inability of the ss-pth2 strains to effectively maintain and use either endogenous nutrient supplies or plant nutrients. Consistent with this possibility, it has been reported that substantial levels of glycerol accumulate in S. sclerotiorum-infected cotyledons (Jobic et al. 2007). These findings suggested that glycerol is specifically localized in fungal hy- 
phae and derived during host colonization by degradation of fungal lipid storage compounds (Jobic et al. 2007).

The restricted lesions produced by the ss-pth2 strain are indicative of the plant having recognized the fungus and initiated a defense response, with consequent containment of the fungus. Recent studies have demonstrated that the function of oxalate produced by WT S. sclerotiorum differs at the different phases of infection. Specifically, oxalate initially generates an environment that suppresses host defenses, to allow pathogen establishment. Once infection is established, oxalate induces production of plant reactive oxygen species that cause host programmed cell death (PCD) (Kim et al. 2008), which is exploited by the fungus to facilitate its proliferation (Jobic et al. 2007; Williams et al. 2011). The idea that PCD plays a pivotal role in the interaction of $S$. sclerotiorum with its host plant is supported by findings that transgenic plants expressing heterologous anti-apoptotic genes have an increased resistance to S. sclerotiorum (Dickman et al. 2001). Although slower in planta growth of the ss-pth2 mutant could explain a reduced lesion expansion rate, it cannot fully account for the observed lesion phenotype. The defense response and restricted lesions suggest that the kinetics of oxalate production by the mutant are not optimal to enable the fungus to override the initial plant defense system and, subsequently, generate a PCD sufficient for full pathogenic success. These findings point to subtleties in the metabolic balance between hosts and this necrotrophic pathogen which influence levels of virulence and resistance.

This study has established several lines of evidence that demonstrate the involvement of the Ss-pth2 carnitine shuttle not only in development of $S$. sclerotiorum reproductive structures but also for oxalate production and virulence of the fungus. Thus, the broad capacity and flexibility of S. sclerotiorum metabolism plays a significant role in the pathogenic success of this necrotroph. Further characterization of regulators of peroxisome function may provide additional targets for understanding the multiple roles of peroxisomes in fungal metabolism and pathogenicity of S. sclerotiorum.

\section{MATERIALS AND METHODS}

\section{Fungal strains and culture conditions.}

WT S. sclerotiorum isolate 274 (provided by M. Boosalis, Department of Plant Pathology, University of Nebraska) was used to generate all strains characterized in this study. Fungal culture maintenance and composition of the MM and CM were as described previously (Liberti et al. 2007). HygB-resistant strains were grown on CM agar amended with $\mathrm{HygB}$ at 100 $\mu \mathrm{g} / \mathrm{ml}$ (Calbiochem, EMD Biosciences, Inc., La Jolla, CA, U.S.A.). Nourseothricin-resistant strains were grown on CM amended with nourseothricin at $100 \mu \mathrm{g} / \mathrm{ml}$ (Werner BioAgents, Jena, Germany).

To initiate growth from fungal stocks, sclerotia were germinated on CM agar. To test the effects of different carbon sources on mycelial growth and pathogenicity, CM-grown colonies were subcultured onto MM-C. Mycelial plugs from actively growing MM-C colonies were then transferred to MM supplemented with glucose at $10 \mathrm{~g} / \mathrm{liter}$ as sole carbon source (MM+Glu), or a molar equivalent amount of the following alternative carbon sources: glycerol $(\mathrm{MM}+\mathrm{Gly})$, potassium acetate (MM+KAc), olive oil (MM+Olive), triolein (MM+Tri), or oleic acid $(\mathrm{MM}+\mathrm{OlAc})$.

YPSu liquid shake cultures $(50 \mathrm{ml})$ were cultured as previously described (Rollins and Dickman 2001) for analysis of oxalate accumulation kinetics. Mycelial inocula for pathogenicity tests were prepared from cultures grown on CM agar or MM agar containing one of the carbon sources listed above.

\section{Ascospore production.}

Carpogenic germination of $S$. sclerotiorum sclerotia was induced using methods similar to those described by Kosasih and Willetts (1975). Sclerotia were produced by following growth on CM agar for 4 weeks at room temperature, then removed from the agar and air dried for 3 to 4 days. The sclerotia were incubated at $-20^{\circ} \mathrm{C}$ for 4 months in sealed containers containing anhydrous calcium sulfate desiccant (W. A. Hammond Drierite Co. Ltd., Xenia, OH, U.S.A.). Conditioned sclerotia were transferred to petri dishes containing $1 \%$ agar (WA), and incubated at $21^{\circ} \mathrm{C}$ with a 12 -h/day exposure to UV-A fluorescent lights (Osram Sylvania Inc., Danvers, MA, U.S.A.). Humidity was maintained by adding $1 \mathrm{ml}$ of sterile water to each petri dish every 3 to 4 days, and sealing the plates with Parafilm. Apothecial initials appeared on the sclerotia surfaces after 3 to 4 weeks. Ascospores were collected aseptically as follows: newly formed, fully expanded apothecia were excised from the sclerotia and inverted overnight onto aluminum foil in a petri dish (Dhingra and Sinclair 1995). The ascospore-containing foil was placed between sterile Whatman filter paper, transferred to coin envelopes, and stored at $-20^{\circ} \mathrm{C}$ over desiccant (W. A. Hammond Drierite Co. Ltd.) and in airtight plastic containers.

\section{Isolation and analysis of nucleic acids.}

For genomic DNA isolation, confluent cultures of S. sclerotiorum were grown on CM agar overlaid with sterile cellophane (Gel Drying Film; Promega Corp., Madison, WI, U.S.A.). The cultures were incubated for 3 days at room temperature and mycelial mats were peeled from the cellophane, transferred to liquid nitrogen, ground to a fine powder, and DNA extracted as described previously (Rollins and Dickman 2001). For isolation of total RNA, cultures were grown on various agar media (CM, PDA, MM-C, MM+Glu, MM+Gly, MM+Olive, MM+ OlAc, MM+KAc). RNA was extracted from ground, frozen tissue with TRIzol Reagent (Invitrogen Canada, Burlington, ON, Canada), according to the manufacturer's instructions.

For DNA blot hydridizations, approximately $0.5 \mu \mathrm{g}$ of genomic DNA was digested with restriction enzymes, fractionated by electrophoresis through $0.8 \%$ agarose gels, transferred by capillary blotting to positively charged nylon membranes (Roche Biomolecular Diagnostics, Laval, Quebec, Canada), and hybridized with Ss-pth2- and $H P H\left(\mathrm{HygB}^{\mathrm{r}}\right.$ gene-specific, digoxigenin [DIG]-labeled gene) amplicons synthesized from plasmid templates using primer pairs Spth2-p3 and Spth2-rev1 or Hyg-1 and Hyg-2 (these and all other primers used in this study and the extension time used with each primer set are listed in Supplementary Table S1). Blots were prepared and hybridized as described previously (Dobinson et al. 2004). To rehybridize the blots with different amplicons, probes were stripped from the blots by incubating for $20 \mathrm{~min}$ at $68^{\circ} \mathrm{C}$ in 0.2 $\mathrm{N} \mathrm{NaOH}$ and $0.1 \%$ sodium dodecyl sulfate.

For Northern hybridization analyses, approximately $5 \mu \mathrm{g}$ of total RNA was separated on $0.8 \%$ agar gels containing formaldehyde (Sambrook and Russell 2001) and transferred by capillary blotting, as described above. Blots were hybridized with DIG-labeled PCR amplicons synthesized from plasmid templates containing $S s-p t h 2, S s-m l s 1, S s$-icll, or $S s$-oahl sequences. Hybridizations and washes were carried out at $65^{\circ} \mathrm{C}$ according to the manufacturer's instructions, and using the DIG Easy Hyb Granules hybridization solution (Roche Biomolecular Diagnostics).

PCR amplifications were performed in $25-\mu 1$ reactions containing 5 to $10 \mathrm{ng}$ of template DNA, 10 pmol of each primer, $0.2 \mathrm{mM}$ dNTPs, $2.5 \mu \mathrm{l}$ of $10 \times$ reaction buffer, $1.5 \mathrm{mM} \mathrm{MgCl}$, and $0.5 \mathrm{U}$ of Platinum Taq (Invitrogen, Carlsbad, CA, U.S.A.). PCR conditions were as follows: initial denaturation at $95^{\circ} \mathrm{C}$ 
for $5 \mathrm{~min} ; 35$ cycles of $95^{\circ} \mathrm{C}$ for $30 \mathrm{~s}, 58^{\circ} \mathrm{C}$ for $30 \mathrm{~s}$, and $72^{\circ} \mathrm{C}$ for 1 to $3 \mathrm{~min}$ (depending on expected amplicon size [1 to 6 $\mathrm{kb}]$ ); final extension at $72^{\circ} \mathrm{C}$ for $10 \mathrm{~min}$. Amplicon quality was assessed on ethidium-bromide-stained $0.8 \%$ agarose gels.

For RT-PCR, RNA $(0.5 \mu \mathrm{g})$ was treated with amplificationgrade DNase I (Invitrogen) and reverse transcribed with SuperScriptII RNAse $\mathrm{H}^{-}$reverse transcriptase and oligo(dT)12-18 primer, according to the supplier's protocols (Invitrogen). PCR amplifications of $S s$-pth2 from the cDNA were done using the aforementioned amplification conditions and following primer pairs: i) Spth2-p1/Spth2-rev5 SsPth2-specific primers which flank the Sspth2 coding region, ii) Spth2-p5/Sspth2-rev2 genespecific primers that flank Sspth2, or iii) Chlor-p1/Chlor-rev1 $\mathrm{CAM}^{\mathrm{r}}$-gene-specific primers. To verify that RNA samples were not contaminated with genomic DNA, DNase-treated RNA samples that had not been reverse transcribed were used as PCR templates.

\section{Identification of Ss-pth2, and construction of fungal transformation vectors.}

The full-length sequence of the $S$. sclerotiorum peroxisomal CAT SS-pth2 (SS1G_13339.1) was retrieved from the S. sclerotiorum genome sequence database (Broad Institute of Harvard and MIT) by performing BlastP with the $M$. grisea Pth2 sequence (accession number AAB88887). The Ss-pth2 coding sequence and intron position were confirmed by sequence analysis of a cDNA amplicon generated by reverse transcription of RNA from the WT S. sclerotiorum strain, and cloned as follows. The cDNA amplicon was purified using the MinElute PCR purification kit (Qiagen Inc., Valencia, CA, U.S.A.) and was cloned into the pGEM-T Easy vector using the System I kit (Promega Corp.) according to the respective manufacturer's instructions, and was then transformed into Escherichia coli XL1-Blue MRF' competent cells (Stratagene, Agilent Technologies Inc., Santa Clara, CA, U.S.A.). The resulting plasmid, pDLspth2-2, was sequenced in both directions at the Southern Crop Protection and Food Research Centre, Agriculture and Agri-Food Canada (London, ON, Canada). Ss-pth2 protein prediction analysis was subsequently performed using ab initio gene prediction software FGENESH (Salamov and Solovyev 2000) running on the Softberry web interface, with $S$. sclerotiorum as the reference genome. Intron-exon identity was ascertained by searching for fungal intron consensus $5^{\prime}$ and $3^{\prime}$ splicing sites (Ballance 1986), and comparison of corresponding cDNA and gDNA sequences.

To generate a gene-disruption vector, primers spth2-P3 and spth2-Rev1 were designed from $S s$-pth2 and used to amplify from strain $274 \mathrm{gDNA}$ a $1.5-\mathrm{kb}$ sequence corresponding to the full-length gene. The amplicon was cloned into pGEM-T Easy (Promega Corp.), and a 1.3-kb SalI-HindIII fragment of the cloned DNA subcloned into a SalI/HindIII-digested binary vector (pDHt) (Mullins et al. 2001). The resulting plasmid (pDLspth2) was subjected to in vitro transposon (Tn) mutagenesis as described previously (Dobinson et al. 2004), using the EZ::TN system (Epicentre Biotechnologies, Madison WI, U.S.A.) and custom vector pSK846, which carries a gene cassette containing the $\mathrm{CAM}^{\mathrm{r}}$ and $\mathrm{Hyg}^{\mathrm{r}}$ genes. pDLspth2Tn5, in which the resistance gene cassette was inserted into the middle of the 1.3-kb Ss-pth2 gene fragment (Fig. 2), was identified by PCR screening using primers Spth2-p4 and Spth2-rev2, and the exact position of the insertion site was determined by sequencing the plasmid using pMOD-2 forward and reverse sequencing primers (Epicentre Biotechnologies).

To construct the Ss-pth2 complementation vector, an Argo Map search of the $S$. sclerotiorum genome database was conducted to identify a fosmid $(8186 \mathrm{H} 4)$ containing the fulllength $S s$-pth2 locus. A $12.4-\mathrm{kb}$ NheI fragment containing $S s$ - pth2 was subcloned from fosmid $8186 \mathrm{H} 4$ into the NheI site of the 4.8-kb pD-NAT1 vector, which contains the nourseothricin resistance gene (Kuck and Hoff 2006) as a selectable marker. The resulting 17.2-kb plasmid, designated pDL103, was used for protoplast transformation of the null-10 mutant (discussed below).

For phylogenetic analyses, BLAST searches were performed against the NCBI and Institut National de la Recherche Agronomique URGI databases. The percent identity and similarity between $S$. sclerotiorum predicted proteins and retrieved GenBank database sequences were calculated using the BLAST/ bl2seq alignment tool (Altschul et al. 1997). Nucleotide and protein sequence alignments were performed using CLUSTAL W (Thompson et al. 1994) and MEGA5 (Tamura et al. 2011). Nucleotide and amino acid percent divergence within and between groups of sequences was calculated using MEGA5 and the neighbor-joining phylogenetic tree inference method. The statistical confidence of tree topology was evaluated based on values for bootstrap $70 \%$ majority-rule consensus for 1,000 replicates.

\section{Fungal transformations.}

pDLspth2-Tn5 was used to generate $S$. sclerotiorum ss-pth2 null mutants by ATMT (Liberti et al. 2007) of strain 274 ascospores. $\mathrm{HygB}^{\mathrm{r}}$ transformants were purified by $\mathrm{HT}$ isolation as described by Liberti and associates (2007) and screened by PCR amplification and DNA blot hybridization to identify null mutants. To generate genetically pure strains, transformants were carpogenically germinated, as described in Supplementary Text. Random ascospores from single excised apothecia were then germinated for 2 days on WA; individual germinating spores were picked from the agar with a 5.0-mm MicroSharp Blade (BD Ophthalmic System, Waltham, MA, U.S.A.); and, because ascospores of transformants did not at the outset grow vigorously when transferred directly to SM (CM plus $\mathrm{HygB}$ at $100 \mu \mathrm{g} / \mathrm{ml}$; data not shown), SA cultures were initially grown on CM agar, then subcultured onto SM to verify $\mathrm{HygB}^{\mathrm{r}}$. To confirm that SA null mutants were free of WT nuclei, cultures were assessed by both DNA blot hybridization and PCR amplification as described above.

For generation of complemented transformants, protoplasts from the ss-pth2 (null-10) mutant were prepared and transformed with circular pDL103, putative transformants were subcultured two to three times on CM amended with nourseothricin at $50 \mu \mathrm{g} / \mathrm{ml}$, and well-established colonies were subjected to at least two sequential HT isolations as described above.

\section{Compound appressoria production and plant infection assays.}

The production of compound appressoria was assayed on PDA overlaid with autoclaved 9-cm-diameter cellophane circles (Bio-Rad Laboratories Inc., Hercules, CA, U.S.A.) and inoculated with agar-plugs (5 $\mathrm{mm}$ in diameter) taken from the margins of PDA-grown cultures. Plates were incubated at room temperature for $48 \mathrm{~h}$ and photographs were taken of the underside of the colonies.

In other compound appressoria production assays, Parafilm or plastic was overlaid onto sterile, water-saturated Whatman number 1 filter paper in petri plates and inoculated with mycelial plugs as above. Onion epidermis infection assays were performed, without chloroform treatment, following the procedure described by Gourgues and associates (2004).

For plant infection assays, soybean (Glycine max (L.) Merr. 'Harosoy') seedlings were grown for approximately 5 weeks in the greenhouse at temperatures between $25^{\circ} \mathrm{C}$ (night) and $35^{\circ} \mathrm{C}$ (day). Individual, fully expanded soybean leaflets were 
excised and placed in 9-cm petri dishes lined with Whatman filter paper as described above. Each leaflet was inoculated with a single, 5-mm plug excised from the margin of a PDAgrown colony, and plates were sealed with Parafilm (Pechiney Plastic Packaging Company, Chicago) to maintain humidity. Each isolate was inoculated onto one leaflet per plate, three replicate plates per isolate were made per experiment, and the experiment was conducted four times. Each leaflet was digitally photographed at $24,48,72,96$, and 120 hpi. The images were imported to the SPOT advanced Software program (Diagnostic Instruments Inc., Sterling Heights, MI, U.S.A.), and lesion area was calculated by tracing the necrotic area and subtracting the inoculum plug area.

\section{Microscopy.}

S. sclerotiorum ascospores were scraped from aluminum filter stocks, resuspended in water to a concentration of $1 \times 10^{4}$ spores $/ \mathrm{ml}$, and inoculated onto cellophane squares overlaid onto CM or MM amended with different carbon sources. Plates were incubated at room temperature and observed at intervals for appressorium formation. Single cellophane squares containing germinating ascospores were mounted directly onto glass cover slips and examined with a compound light microscope (model DM R HC; Leica, Wetzlar, Germany).

\section{In vitro and in vivo oxalic acid accumulation assays.}

Oxalate accumulation was assayed as described by Rollins and Dickman (2001). Supernatants from cultures grown in MM containing molar equivalent amounts of different carbon sources (Glu, Gly, OlAc, or KAc), and buffered at pH 7 were analyzed for oxalic acid content with an enzyme-based assay kit (Trinity Biotech, St. Louis) according to the manufacturer's instructions. The oxalic acid concentration was determined by comparison with a standard curve. The mean and standard deviation of three replications were adjusted by background subtraction.

For analysis of in planta oxalate production, 5-mm-diameter plugs were taken at 4 days postinoculation with a cork borer from the necrotic potion of the soybean lesion margins and lyophilized; and, using a pipette tip, the dried tissue was homogenized in $25 \mu$ of deionized water. Oxalate concentrations were measured as above.

\section{ACKNOWLEDGMENTS}

We thank U. Benny and S. Grant for technical assistance. Research at the University of Florida was supported through CRIS project FLA-PLP004739. Research at Agriculture and Agri-Food Canada-London was supported by AAFC genomics and A-base program funding.

\section{LITERATURE CITED}

Altschul, S. F., Madden, T. L., Schäffer, A. A., Zhang, J., Zhang, Z., Miller, W. and Lipman, D. J. 1997. Gapped BLAST and PSI-BLAST: A new generation of protein database search programs. Nucleic Acids Res. 25:3389-3402.

Amor, C., Domínguez, A. I., De Lucas, J. R. and Laborda, F. 2000. The catabolite inactivation of Aspergillus nidulans isocitrate lyase occurs by specific autophagy of peroxisomes. Arch. Microbiol. 174:59-66.

Amselem, J., Cuomo, C. A., van Kan, J. A. L., Viaud, M., Benito, E. P., Couloux, A., Coutinho, P. M., de Vries, R. P., Dyer, P. S., Fillinger, S., Fournier, E., Gout, L., Hahn, M., Kohn, L., Lapalu, N., Plummer, K. M., Pradier, J. M., Quévillon, E., Sharon, A., Simon, A., Have, A., Tudzynski, B., Tudzynski, P., Wincker, P., Andrew, M., Anthouard, V., Beever, R. E., Beffa, R., Benoit, I., Bouzid, O., Brault, B., Chen, Z., Choquer, M., Collémare, J., Cotton, P., Danchin, E. G., Da Silva, C., Gautier, A., Giraud, C., Giraud, T., Gonzalez, C., Grossetete, S., Güldener, U., Henrissat, B., Howlett, B. J., Kodira, C., Kretschmer, M., Lappartient, A., Leroch, M., Levis, C., Mauceli, E., Neuvéglise, C., Oeser, B., Pearson, M., Poulain, J., Poussereau, N., Quesneville, H., Rascle, C., Schumacher,
J., Ségurens, B., Sexton, A., Silva, E., Sirven, C., Soanes, D. M., Talbot, N. J., Templeton, M., Yandava, C., Yarden, O., Zeng, Q., Rollins, J. A., Lebrun, M. H. and Dickman, M. 2011. Genomic analysis of the necrotrophic fungal pathogens Sclerotinia sclerotiorum and Botrytis cinerea. PLoS Genet. 7:e1002230. Published online.

Asakura, M., Okuno, T., and Takano, Y. 2006. Multiple contributions of peroxisomal metabolic function to fungal pathogenicity in Colletotrichum lagenarium. Appl. Environ. Microbiol. 72:6345-6354.

Ballance, D. J. 1986. Sequences important for gene expression in filamentous fungi. Yeast 2:229-236.

Bhambra, G. K., Wang, Z. Y., Soanes, D. M., Wakley, G. E., and Talbot, N. J. 2006. Peroxisomal carnitine acetyl transferase is required for elaboration of penetration hyphae during plant infection by Magnaporthe grisea. Mol. Microbiol. 61:46-60.

Boland, G. J., and Hall, R. 1994. Index of plant hosts of Sclerotinia sclerotiorum. Can. J. Plant Pathol. 16:93-108.

Bolton, M. D., Thomma, B. P. H. J., and Nelson, B. D. 2006. Sclerotinia sclerotiorum (Lib.) de Bary: Biology and molecular traits of a cosmopolitan pathogen. Mol. Plant Pathol. 7:1-16.

Cessna, S. G., Sears, V. E., Dickman, M. B., and Low, P. S. 2000. Oxalic acid, a pathogenicity factor for Sclerotinia sclerotiorum, suppresses the oxidative burst of the host plant. Plant Cell 12:2191-2199.

Coley-Smith, J. R., and Cooke, R. C. 1971. Survival and germination of fungal sclerotia. Annu. Rev. Phytopathol. 9:65-92.

Culbertson, B. J., Krone, J., Gatebe, E., Furumo, N. C., and Daniel, S. L. 2007. Impact of carbon sources on growth and oxalate synthesis by the phytopathogenic fungus Sclerotinia sclerotiorum. World J. Microbiol. Biotechnol. 23:1357-1362.

de Jong, J. C., McCormack, B. J., Smirnoff, N., and Talbot, N. J. 1997. Glycerol generates turgor in rice blast. Nature 389:244-245.

De Lucas, J. R., Valenciano, S., Laborda, F., and Turner, G. 1994. Glucose-induced inactivation of isocitrate lyase in Aspergillus nidulans. Arch. Microbiol. 162:409-413.

Dhingra, O. D., and Sinclair, J. B. 1995. Basic Plant Pathology Methods, 2nd ed. CRC Press Inc., Boca Raton, FL. U.S.A

Dickman, M. B., Park, Y. K., Oltersdorf, T., Li, W., Clemente, T., and French, R. 2001. Abrogation of disease development in plants expressing animal antiapoptotic genes. Proc. Natl. Acad. Sci. U.S.A. 98:69576962.

Dobinson, K. F., Grant, S. J., and Kang, S. 2004. Cloning and targeted disruption, via Agrobacterium tumefaciens-mediated transformation, of a trypsin protease gene from the vascular wilt fungus Verticillium dahliae. Curr. Genet. 45:104-110.

Dutton, M. V., and Evans, C. S. 1996. Oxalate production by fungi: Its role in pathogenicity and ecology in the soil environment. Can. J. Microbiol. 42:881-895.

Godoy, G., Steadman, J., Dickman, M., and Dam, R. 1990. Use of mutants to demonstrate the role of oxalic acid in pathogenicity of Sclerotinia sclerotiorum on Phaseolus vulgaris. Physiol. Mol. Plant Pathol. 37:179191.

Gourgues, M., Brunet-Simon, A., Lebrun, M. H., and Levis, C. 2004. The tetraspanin BcPIs1 is required for appressorium-mediated penetration of Botrytis cinerea into host plant leaves. Mol. Microbiol. 51:619-629.

Idnurm, A., Giles, S. S., Perfect, J. R., and Heitman, J. 2007. Peroxisome function regulates growth on glucose in the basidiomycete fungus Cryptococcus neoformans. Eukaryotic Cell 6:60-72.

Jobic, C., Boisson, A. M., Gout, E., Rascle, C., Fèvre, M., Cotton, P., and Bligny, R. 2007. Metabolic processes and carbon nutrient exchanges between host and pathogen sustain the disease development during sunflower infection by Sclerotinia sclerotiorum. Planta 226:251-265.

Kim, K. S., Min, J. Y., and Dickman, M. B. 2008. Oxalic acid is an elicitor of plant programmed cell death during Sclerotinia sclerotiorum disease development. Mol. Plant-Microbe Interact. 21:605-612.

Kosasih, B. D., and Willetts, H. J. 1975. Ontogenetic and histochemical studies of the apothecium of Sclerotinia sclerotiorum. Ann. Bot. 39:185-191.

Kuck, U., and Hoff, B. 2006. Application of the nourseothricin acetyltransferase gene (natl) as dominant marker for the transformation of filamentous fungi. Fungal Genet. Newsl. 53:9.

Kunau, W.-H., Dommes, V., and Schulz, H. 1995. (beta)-Oxidation of fatty acids in mitochondria, peroxisomes, and bacteria: A century of continued progress. Prog. Lipid Res. 34:267-342.

Kurihara, T., Ueda, M., Kanayama, N., Kondo, J., Teranishi, Y., and Tanaka, A. 1992. Peroxisomal acetoacetyl-CoA thiolase of an n-alkaneutilizing yeast, Candida tropicalis. Eur. J. Biochem. 210:999-1005.

Liberti, D., Grant, S. J., Benny, U., Rollins, J. A., and Dobinson, K. F. 2007. Development of an Agrobacterium tumefaciens-mediated gene disruption method for Sclerotinia sclerotiorum. Can. J. Plant Pathol. 29:1-7.

López, M. L., Redruello, B., Valdés, E., Moreno, F., Heinisch, J. J., and 
Rodicio, R. 2004. Isocitrate lyase of the yeast Kluyveromyces lactis in subject to glucose repression but not to catabolite inactivation. Curr. Genet. 44:305-316.

Lorenz, M. C., and Fink, G. R. 2001. The glyoxylate cycle is required for fungal virulence. Nature 412:83-86.

Lösel, D. M., and Sancholle, M. 1996. Fungal lipids. Pages 27-62 in: Lipids of Pathogenic Fungi. P. Rajendra and M. A. Ghannoum, eds. CRC Press, Inc., Boca Raton, FL, U.S.A

Lumsden, R. D., and Dow, R. L. 1973. Histopathology of Sclerotinia sclerotiorum infection of bean. Phytopathology 63:708-715.

Maxwell, D. P. 1973. Oxalate formation in Whetzelinia sclerotiorum by oxaloacetate acetylhydrolase. Physiol. Plant Pathol. 3:279-288.

Maxwell, D. P., and Lumsden, R. D. 1970. Oxalic acid production by Sclerotinia sclerotiorum in infected bean and in culture. Phytopathology 60:1395-1398.

Mullins, E., Chen, X., Romaine, P., Raina, R., Geiser, D., and Kang, S. 2001. Agrobacterium-mediated transformation of Fusarium oxysporum: An efficient tool for insertional mutagenesis and gene transfer. Phytopathology 91:173-180.

Munir, E., Yoon, J. J., Tokimatsu, T., Hattori, T., and Shimada, M. 2001. New role for glyoxylate cycle enzymes in wood-rotting basidiomycetes in relation to biosynthesis of oxalic acid. J. Wood Sci. 47:368-373.

Noverr, M. C., Erb-Downward, J. R., and Huffnagle, G. B. 2003. Production of eicosanoids and other oxylipins by pathogenic eukaryotic microbes. Clin. Microbiol. Rev. 16:517-533.

Purdy, L. H. 1958. Some factors affecting penetration and infection by Sclerotinia sclerotiorum. Phytopathology 48:605-609.

Ramos-Pamplona, M., and Naqvi, N. I. 2006. Host invasion during riceblast disease requires carnitine-dependent transport of peroxisomal acetyl-CoA. Mol. Microbiol. 61:61-75.

Rollins, J. A. 2003. The Sclerotinia sclerotiorum pac1 gene is required for sclerotial development and virulence. Mol. Plant-Microbe Interact 16:785-795

Rollins, J. A., and Dickman, M. B. 2001. pH signaling in Sclerotinia sclerotiorum: Identification of a pacC/RIM1 homolog. Appl. Environ. Microbiol. 67:75-81.

Sakai, S., Nishide, T., Munir, E., Baba, K., Inui, H., Nakano, Y., Hattori, T., and Shimada, M. 2006. Subcellular localization of glyoxylate cycle key enzymes involved in oxalate biosynthesis of wood-destroying basidiomycete Fomitopsis palustris grown on glucose. Microbiology 152:1857-1866.

Salamov, A. A., and Solovyev, V. V. 2000. Ab initio gene finding in Drosophila genomic DNA. Genome Res. 10:516-522.

Sambrook, J., and Russell, D. W. 2001. Molecular Cloning: A Laboratory Manual. Cold Spring Harbor Laboratory Press, Cold Spring Harbor, NY, U.S.A.

Smith, J. J., Brown, T. W., Eitzen, G. A., and Rachubinski, R. A. 2000. Regulation of peroxisome size and number by fatty acid $\beta$-oxidation in the yeast Yarrowia lipolytica. J. Biol. Chem. 275:20168-20178.
Solomon, P. S., Tan, K. C., and Oliver, R. P. 2003. The nutrient supply of pathogenic fungi; a fertile field for study. Mol. Plant Pathol. 4:203-210.

Solomon, P. S., Lee, R. C., Wilson, T. J., and Oliver, R. P. 2004. Pathogenicity of Stagonospora nodorum requires malate synthase. Mol. Microbiol. 53:1065-1073.

Stemple, C. J., Davis, M. A., and Hynes, M. J. 1998. The facC gene of Aspergillus nidulans encodes an acetate-inducible carnitine acetyltransferase. J. Bacteriol. 180:6242-6251.

Strijbis, K., Van Roermund, C. W. T., Visser, W. F., Mol, E. C., Van Den Burg, J., MacCallum, D. M., Odds, F. C., Paramonova, E., Krom, B. P., and Distel, B. 2008. Carnitine-dependent transport of acetyl coenzyme A in Candida albicans is essential for growth on nonfermentable carbon sources and contributes to biofilm formation. Eukaryotic Cell 7:610-618.

Strijbis, K., Van Roermund, C. W., Van Den Burg, J., Van Den Berg, M., Hardy, G. P. M., Wanders, R. J., and Distel, B. 2010. Contributions of carnitine acetyltransferases to intracellular acetyl unit transport in Candida albicans. J. Biol. Chem. 285:24335-24346.

Tamura, K., Peterson, D., Peterson, N., Stecher, G., Nei, M., and Kumar, S. 2011. MEGA5: Molecular evolutionary genetics analysis using maximum likelihood, evolutionary distance, and maximum parsimony methods. Mol. Biol. Evol. 28:2731-2739.

Tariq, V. N., and Jeffries, P. 1984. Appressorium formation by Sclerotinia sclerotiorum: Scanning electron microscopy. Trans. Br. Mycol. Soc. 82:645-651.

Thines, E., Eilbert, F., Sterner, O., and Anke, H. 1997. Signal transduction leading to appressorium formation in germinating conidia of Magnaporthe grisea: Effects of second messengers diacylglycerols, ceramides and sphingomyelin. FEMS (Fed. Eur. Microbiol. Soc.) Microbiol. Lett. 156:91-94.

Thompson, J. D., Higgins, D. G., and Gibson, T. J. 1994. CLUSTAL W: Improving the sensitivity of progressive multiple sequence alignment through sequence weighting, position-specific gap penalties and weight matrix choice. Nucleic Acids Res. 22:4673-4680.

Wang, Z. Y., Thornton, C. R., Kershaw, M. J., Debao, L., and Talbot, N. J. 2003. The glyoxylate cycle is required for temporal regulation of virulence by the plant pathogenic fungus Magnaporthe grisea. Mol. Microbiol. 47:1601-1612.

Williams, B., Kabbage, M., Kim, H. J., Britt, R., and Dickman, M. B. 2011. Tipping the balance: Sclerotinia sclerotiorum secreted oxalic acid suppresses host defenses by manipulating the host redox environment. PLoS Pathol. 7:e1002107. Published online.

\section{AUTHOR-RECOMMENDED INTERNET RESOURCES}

Broad Institute of Harvard and MIT: www.broad.mit.edu

NCBI database: www.ncbi.nlm.nih.gov

Softberry web interface: www.softberry.com 